\title{
Blind drunk: alcoholic pancreatitis and loss of vision
}

\author{
James R. Steel, John R. Cockcroft and James M. Ritter
}

\section{Department of Clinical Pharmacology, Guy's Hospital Medical School, London SE1 9RT, UK}

\begin{abstract}
Summary: Acute loss of vision and alcoholic intoxication suggests the possibility of methanol poisoning. In this report we describe an alcoholic patient who complained of blindness after recovering from alcoholic pancreatitis and delirium tremens. Visual acuity was severely impaired and fundoscopy revealed florid bilateral cotton wool spots and a cherry red spot at the right macula. Such appearances have previously been reported in cases of post-traumatic visual loss, and may share a common aetiology of fat embolization. The association between alcoholic pancreatitis and visual loss is rare, and fundal examination should be performed on all patients with pancreatitis, especially those who complain of visual disturbance.
\end{abstract}

\section{Introduction}

Previous case reports have confirmed an association between severe alcoholic pancreatitis and visual loss. ${ }^{1,2}$ These changes are remarkably similar to those seen in post-traumatic retinopathy. Although blindness associated with alcohol abuse suggests the possibility of methanol ingestion, it is important to consider the possibility of a retinopathy due to alcoholic pancreatitis and fundal examination should be performed on all patients with pancreatitis who complain of visual disturbance.

\section{Case report}

A 29 year old man was brought to the casualty department by his wife because he was delirious. She reported that he usually drank alcohol heavily but had almost completely stopped 4 days before, because of vomiting, diarrhoea and abdominal pain. Two days before presenting to casualty he had been well enough to go to his doctor's surgery, but he had subsequently become worse and had been delirious for the past 24 hours. On examination he was acutely unwell, agitated, sweating and experiencing visual hallucinations. His temperature was $37.5^{\circ} \mathrm{C}$, blood pressure $140 / 80 \mathrm{mmHg}$ and pulse $160 /$ minute and regular. No source of infection was evident, his abdomen was soft, there was no neck stiffness or focal neurological signs. However, although his pupils were widely dilated, it was not possible to examine his fundi because of his

Correspondence: Professor J.M. Ritter, F.R.C.P., Department of Clinical Pharmacology, UMDS, Medical School Building, Guy's Hospital, London Bridge, SE1 9RT, UK.

Accepted: 14 August 1992 agitated state. Blood ethanol was $63.8 \mathrm{mg} / 100 \mathrm{ml}$, $\gamma$-glutamyl transpeptidase was raised at $206 \mathrm{U} / 1$ (normal range 11-54), aspartate transaminase raised at $46 \mathrm{U} / 1$ (normal range $<23$ ) and amylase raised at $809 \mathrm{U} / 1$ (normal range $<220$ ). There was a neutrophil leucocytosis with total white cell count of $17.1 \times 10^{9} / 1$ and the mean corpuscular volume was $102 \mathrm{fl}$ (normal range 76-96). Plasma creatinine, glucose and electrolyte concentrations were within normal limits.

A diagnosis of acute pancreatitis complicated by delirium tremens was made, and he was treated with parenteral vitamins, sedated with intravenous chlormethiazole and rehydrated with physiological saline. The delirium resolved, his temperature returned to normal and at no time was the blood pressure greater than the admission reading. The following day he was orientated and rational, and complained that he was 'completely blind'. He explained that his reason for consulting his general practitioner had been because of blurring of vision. He denied drinking methylated spirits. On examination he could perceive light and hand movements with each eye, but could not count fingers. The pupillary light reflex was present and fundoscopy revealed florid bilateral cotton wool spots with a cherry red spot at the right macula (Figure 1). He admitted to having abused drugs up to 2 years previously and had shared needles. However, a test for human immunodeficiency virus was negative and cerebrospinal fluid examination, including tests for cytomegalovirus and fungal infection, was normal. Although the retinal appearances were most similar to those of accelerated hypertension his blood pressure had remained normal throughout. Antinuclear antibodies were sought, because systemic lupus erythematosus can cause cotton wool spots despite normal blood pressure, 


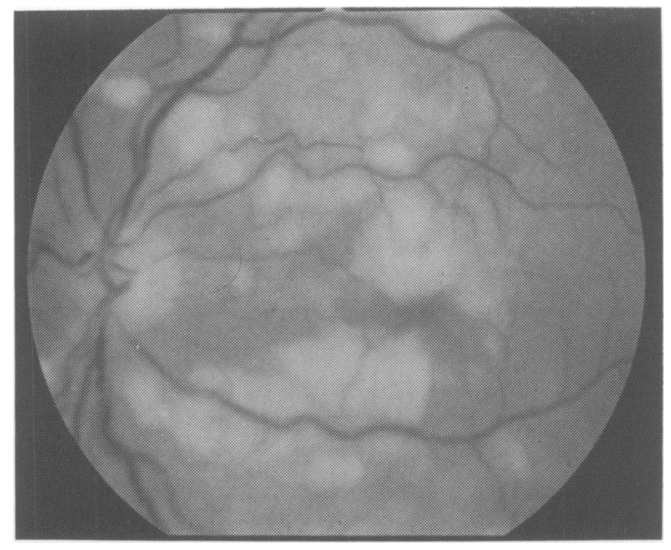

Figure 1 Retinal appearances in a patient with alcoholic pancreatitis. Florid cotton wool spots are present with a cherry red spot at the macula.

but were not present. Ultrasound of his abdomen revealed an enlarged pancreas.

Pancreatitis is, rarely, associated with cotton wool spots and, in the absence of other causes of retinopathy, we believe this to have been the likely explanation of the retinal appearances in our patient. His vision had improved only minimally when he was reviewed a month later as an outpatient.

\section{Discussion}

In 1910 Purtscher described a number of cases of post-traumatic visual loss associated with characteristic retinal changes. ${ }^{3}$ Blindness typically occurred within hours of trauma to the head or body and was accompanied by the appearance of multiple cotton wool spots and haemorrhages. The retinopathy was initially attributed to sudden increases in cerebrospinal fluid pressure producing lymphatic extravasation. Subsequently a clinicopathological correlation between traumatic retinopathy and fat embolization has been made with fat being demonstrated in both the retinal and choroidal vessels of patients developing visual loss following long bone fractures. ${ }^{4}$ Extensive fat embolization to vital organs including brain, lungs, heart and kidneys has also been observed in patients dying with severe pancreatitis. ${ }^{5,6}$ Inkeles and Walsh ${ }^{7}$ reported an association between retinopathy and acute pancreatitis and commented on the marked similarity of the fundal appearances of their patients to those originally described by Purtscher, writing that: 'The retina has multiple, often confluent, cotton wool patches located primarily within the temporal retinal vascular arcades and macula, retinal oedema, and occasionally striate and blot haemorrhages.' They suggested that fat embolization caused both post-traumatic and pancreatitic retinopathy. Subsequent case reports have confirmed both the clinicopathological association between alcoholic pancreatitis and severe visual loss and also the similarity of the retinal changes found to those seen in post-traumatic retinopathy, ${ }^{1,2}$ but the association is still not widely known.

The aetiology of pancreatitic retinopathy remains uncertain, some investigators suggesting that granulocyte aggregates rather than fat emobli are responsible for the retinal arteriolar occlusions. During the course of acute pancreatitis activated proteases such as trypsin are released and activate components of the complement system (including C5) that aggregate granulocytes: serum from patients with acute pancreatitis but not serum from convalescent patients causes granulocyte aggregation. ${ }^{8}$ It has been suggested that such aggregates could cause retinal vascular occlusions. The diameter of these aggregates $(60 \mu \mathrm{m})$ is similar to that of glass spheres $(15-40 \mu \mathrm{m})$ which are needed experimentally to produce an animal model of such retinopathy. ${ }^{9}$

Treatment of retinopathy related to pancreatitis is conservative and essentially limited to observation and supportive care. The visual outcome depends on a number of factors including the pre-existing state of the retinal vasculature, the location of the cotton wool spots and the duration and severity of the vascular compromise.

\section{References}

1. Kincaid, M.C., Green, W.R., Knox, D.L. \& Mohler, C. A clinico-pathological case report of retinopathy of pancreatitis. Br J Ophthalmol 1982, 66: 219-226.

2. Snady-McCoy, L. \& Morse, P.H. Retinopathy associated with acute pancreatitis. Am J Ophthalmol 1985, 100: 246-251.

3. Purtscher, $O$. Noch unbekannte befunde nach Schädeltrauma. Ber Dtsch Ophthalmol Ges 1910, 36: 294-307.

4. Urbanek, J. Uber fettembolie des auges. Arch Klin Ophthalmol 1934, 131: 147-173.

5. Edmondson, H.A. \& Field, I.A. Relation of calcium and lipids to acute pancreatic necrosis. Arch Intern Med 1942, 69: 177-181.

6. Lynch, M.J. Nephrosis and fat embolism in acute hemorrhage pancreatitis. Arch Intern Med 1954, 94: 709-713.

7. Inkeles, D.M. \& Walsh, J.B. Retinal fat emboli as a sequela to acute pancreatitis. Am J Ophthalmol 1975, 80: 935-938.

8. Jacob, H.S., Goldstein, I.M., Shapiro, I., Craddock, P.R., Hammerschmidt, D.E. \& Weissmann, G. Sudden blindness in acute pancreatitis possible role of complement-induced retinal leukoemobilization. Arch Intern Med 1981, 141: 134-136.

9. Ashton, N. \& Henkind, P. Experimental occlusion of retinal arterioles. Br J Ophthalmol 1965, 49: 225-234. 